\title{
The effect of shear tractions on frictionally-excited thermoelastic instability
}

\author{
Kwangin Lee and J. R. Barber \\ Department of Mechanical Engineering and Applied Mechanics, University of Michigan, Ann Arbor, MI 48109-2125 (USA)
}

(Received February 13, 1992; revised and accepted July 9, 1992)

\begin{abstract}
A parametric study is conducted to determine the effect of normal displacements due to shear tractions on the critical speed for frictionally-excited thermoelastic instability. The results show that shear effects can be very significant when the materials have comparable thermal properties and their neglect can lead to a non-conservative overestimate of critical speed. It is also found that the common approximation of one material as a non-conductor typically underestimates the critical speed by an order of magnitude if the ratio of the two material conductivities is greater than 0.005 .
\end{abstract}

\section{Introduction}

If the nominally uniform contact pressure between two sliding bodies is perturbed, non-uniform frictional heat will be generated at the interface and a nonuniform temperature field results. The consequent thermoelastic distortion of the contacting bodies in turn modifies the contact pressure distribution. This feedback process is generally found to be unstable and is called thermoelastic instability (TEI) $[1,2]$. This leads to the development of regions of high local temperature known as hot spots with the consequent danger of material degradation. Experimental evidence of such high temperatures has been observed in many practical applications such as brakes and seals as well as in the laboratory [2-7].

The stability of thermoelastic sliding of conforming solids was examined by Burton and coworkers $[8,9]$. They showed that the contact between two sliding halfplanes is unstable if and only if the sliding speed exceeds a certain critical value, which depends upon the wavelength of the perturbation.

The tangential frictional traction, which gives rise to the generation of heat and hence to the instability, will also tend to produce an elastic normal displacement at the surface which modifies the contact pressure distribution. However, this displacement is generally small compared with the displacement due to the normal tractions and was neglected in Burton's analysis. This simplification is frequently made in thermoelastic calculations [10] and some justification for it can be found in the results of Heckmann and Burton [11] who showed that, for the case of aluminum sliding against a rigid non-conducting plane, the introduction of shear effects only changes the predicted critical speed by $1 \%$. However, we shall show in this work that substantially larger errors may be introduced by neglecting shear effects when both materials are thermal conductors.

\section{Formulation}

The analysis is a routine extension of that of Heckmann and Burton [11] and only the essential steps will be given here. We consider the problem of two halfplanes, $y>0, y<0$ respectively, in sliding contact at the interface $y=0$ and pressed together by a uniform pressure $p_{0}$. The two half-planes are generally of dissimilar materials, appropriate properties being distinguished by the suffix 1 for $y>0$ and 2 for $y<0$. We assume that the bodies have absolute velocities $V_{i}(i=1,2)$ in the $x$-direction and that a perturbation develops in the uniform fields which has absolute velocity $c$ and relative velocity $c_{i}$ with respect to body $i$. It follows that $c=V_{1}+c_{1}=V_{2}+c_{2}$ and that the sliding velocity, $V$, can be written

$V=V_{2}-V_{1}=c_{1}-c_{2}$

The stability of the system can be examined by finding the condition under which a small sinusoidal perturbation in the temperature and stress fields can grow with time. As long as the perturbation is sufficiently small, the half-planes will remain in contact throughout the interface. The temperature perturbations in each 
body which satisfy the transient heat conduction equation can be written in a frame of reference stationary with respect to the perturbation as

$T_{1}=\mathfrak{R}\left\{T_{0} \exp \left(b t-\lambda_{1} y+j m x\right)\right\}$

$T_{2}=\mathfrak{R}\left\{T_{0} \exp \left(b t+\lambda_{2} y+j m x\right)\right\}$

where $j=\sqrt{-1}$ and $T_{0}$ is an arbitrary constant

$\lambda_{i} \equiv \xi_{i}+j \eta_{i}$

$\xi_{i}=\left\{\frac{1}{2}\left[\left(m^{2}+\frac{b}{k_{i}}\right)+\sqrt{\left(m^{2}+\frac{b}{k_{i}}\right)^{2}+\left(\frac{m c_{i}}{k_{i}}\right)^{2}}\right]\right\}^{1 / 2}$

$\eta_{i}=(-1)^{i}\left\{\frac{1}{2}\left[-\left(m^{2}+\frac{b}{k_{i}}\right)+\sqrt{\left(m^{2}+\frac{b}{k_{i}}\right)^{2}+\left(\frac{m c_{i}}{k_{i}}\right)^{2}}\right]\right\}^{1 / 2}$

and $k_{i}$ is the thermal diffusivity of body $i$. The thermoelastic stress and displacement fields corresponding to the temperature distribution (eqns. (2) and (3)) are then determined, the resulting arbitrary constants being assigned so as to satisfy the frictional contact boundary conditions:

$u_{y 1}=u_{y 2} \quad y=0$

$\sigma_{y y 1}=\sigma_{y y 2} \quad y=0$

$\sigma_{x y i}=f \sigma_{y y i} \quad y=0$

where $f$ is the coefficient of friction and eqn. (9) is appropriate for the case where $V>0$. Equations (2) and (3) satisfy the requirement of continuity of temperature at the sliding interface and it remains to satisfy the condition that the rate of heat generation at the interface due to the frictional tractions is equal to the rate of conduction away from the interface, i.e.

$q_{\mathrm{net}}=q_{y 1}-q_{y 2}=f V p \quad y=0$

which leads to the complex characteristic equation

$$
\begin{aligned}
& \left(K_{1} \lambda_{1}+K_{2} \lambda_{2}\right)(1-j f \beta)=\frac{2 m f V}{\left(\frac{1-\nu_{1}}{\mu_{1}}+\frac{1-\nu_{2}}{\mu_{2}}\right)} \\
& \quad \times\left\{\frac{\alpha_{1}\left(1+\nu_{1}\right)}{\lambda_{1}+m}+\frac{\alpha_{2}\left(1+\nu_{2}\right)}{\lambda_{2}+m}\right\}
\end{aligned}
$$

where $K, \alpha, \mu, \nu$ are thermal conductivity, heat expansion coefficient, modulus of rigidity, Poisson's ratio respectively and

$\beta=\frac{1}{2}\left\{\frac{\mu_{2}\left(1-2 \nu_{1}\right)-\mu_{1}\left(1-2 \nu_{2}\right)}{\mu_{2}\left(1-\nu_{1}\right)+\mu_{1}\left(1-\nu_{2}\right)}\right\}$

is Dundurs' constant [12]. In elastic contact problems, $\beta$ can be interpreted as a dimensionless measure of the coupling between the normal and tangential loading problems (see Chapter 12 of ref. 13).

The characteristic eqn. (11) serves to determine the exponential growth rate, $b$, for a disturbance of given spatial frequency, $m$, and sliding speed, $V$. We note that the coupling between normal and tangential tractions, which forms the subject of the present paper, is proportional to Dundurs' constant and enters the characteristic equation through the term $f \beta$. The characteristic eqn. (11) can be restated in the dimensionless form

$$
\left(\lambda_{1}^{*}+K^{*} \lambda_{2}^{*}\right)(1-j f \beta)=f V^{*} H_{1}\left\{\frac{1}{\lambda_{1}^{*}+1}+\alpha^{*} \frac{1}{\lambda_{2}^{*}+1}\right\}
$$

where

$$
\begin{aligned}
K^{*} & =\frac{K_{2}}{K_{1}} ; k^{*}=\frac{k_{2}}{k_{1}} ; \alpha^{*}=\frac{\alpha_{2}\left(1+\nu_{2}\right)}{\alpha_{1}\left(1+\nu_{1}\right)} \\
\lambda_{i}^{*} & =\frac{\lambda_{i}}{m} ; c_{i}^{*}=\frac{c_{i}}{k_{1} m} \\
V^{*} & =c_{1}^{*}-c_{2}^{*} \\
H_{1} & =\frac{2 \alpha_{1}\left(1+\nu_{1}\right) k_{1}}{K_{1}\left(\frac{1-\nu_{1}}{\mu_{1}}+\frac{1-\nu_{2}}{\mu_{2}}\right)}
\end{aligned}
$$

Notice that the dimensionless parameter, $H_{1}$, differs from the thermomechanical material parameter, $H$, introduced by Hills and Barber [14] in that a composite elastic compliance appears in the denominator. However, if material 2 were rigid $\left(\mu_{2} \rightarrow \infty\right), H_{1}$ would reduce to the value of $H$ for material 1 . Hills and Barber showed that $H$ is close to unity for a wide range of practical materials.

\subsection{The stability boundany}

Perturbations will generally only be possible for certain eigenvalues of the exponential growth rate, $b$. Stability will be maintained if the growth rates of all such perturbations are negative since all initial perturbations will then decay with time. Thus we can find the stability boundary by setting the growth rate to zero and hence obtain the critical sliding speed which depends upon the wavelength of the perturbation. When $b=0$, the dimensionless forms of eqns. (4-6) reduce to

$\lambda_{i}^{*}=\xi_{i}^{*}+j \eta_{i}^{*}$

$\xi_{1}^{*}=\left\{\frac{1}{2}\left[1+\sqrt{1+\left(c_{1}^{*}\right)^{2}}\right]\right\}^{1 / 2}$ 
$\eta_{1}^{*}=-\left\{\frac{1}{2}\left[-1+\sqrt{1+\left(c_{1}^{*}\right)^{2}}\right]\right\}^{1 / 2}$

$\xi_{2}^{*}=\left\{\frac{1}{2}\left[1+\sqrt{1+\left(\frac{c_{2}^{*}}{k^{*}}\right)^{2}}\right]\right\}^{1 / 2}$

$\eta_{2}^{*}=\left\{\frac{1}{2}\left[-1+\sqrt{1+\left(\frac{c_{2}^{*}}{k^{*}}\right)^{2}}\right]\right\}^{1 / 2}$

which imply the relations

$\xi_{1}^{* 2}-\eta_{1}^{* 2}=1 ; \xi_{2}^{* 2}-\eta_{2}^{* 2}=1$

After substituting eqn. (18) into eqn. (13) and separating the real and imaginary parts, we obtain two real equations, which, using eqn. (23) can be written

$$
\begin{aligned}
& \left(\xi_{1}^{*}+K^{*} \xi_{2}^{*}\right)+f \beta\left(\eta_{1}^{*}+K^{*} \eta_{2}^{*}\right)=\frac{f H_{1} V^{*}}{2}\left(\frac{1}{\xi_{1}^{*}}+\frac{\alpha^{*}}{\xi_{2}^{*}}\right) \\
& \left(\eta_{1}^{*}+K^{*} \eta_{2}^{*}\right)-f \beta\left(\xi_{1}^{*}+K^{*} \xi_{2}^{*}\right) \\
& =-\frac{f H_{1} V^{*}}{2}\left\{\frac{\left(\xi_{1}^{*}-1\right)}{\xi_{1}^{*} \eta_{1}^{*}}+\alpha^{*} \frac{\left(\xi_{2}^{*}-1\right)}{\xi_{2}^{*} \eta_{2}^{*}}\right\}
\end{aligned}
$$

These equations and relation (16) can be used to eliminate $c_{1}^{*}, c_{2}^{*}$ and hence determine the dimensionless critical speed $V^{*}$.

\subsection{Solutions when one body is a non-conductor}

The limiting case where one of the bodies is a nonconductor was treated by Heckmann and Burton [11]. The equations for this case can be obtained by a similar process from first principles or alternatively from the more general solution by a limiting process. Taking the non-conducting body to be body 2 , we obtain

$\xi_{1}^{*}+f \beta \eta_{1}^{*}=\frac{f H_{1} V^{*}}{2 \xi_{1}^{*}} ; \eta_{1}^{*}-f \beta \xi_{1}^{*}=-\frac{f H_{1} V^{*}\left(\xi_{1}^{*}-1\right)}{2 \xi_{1}^{*} \eta_{1}^{*}}$

When $\beta=0$, these equations have the simple solution

$V_{0}^{*}=\frac{2}{f H_{1}}$

\section{Results}

The determination of the critical speed is clearly considerably simplified if shear effects are neglected - i.e. if $\beta$ is set to equal to zero - and hence the principal motivation for the present work is to determine in what ranges of the parameters this simplification gives a reasonable approximation to the exact result and, more generally, what percentage error is to be anticipated from its use.

\subsection{One material non-conducting}

This question can be answered with some generality for the case where one material is a non-conductor, since eqns. (26) contain only two independent dimensionless parameters $f H_{1}$ and $f \beta$. Furthermore, $f H_{1}$ and $V^{*}$ only occur in the combination $f H_{1} V^{*}$ and hence a unique relationship can be found between this combination and $f \beta$. In view of eqn. (27), $f H_{1} V^{*} / 2=V^{*} /$ $V_{0}^{*}$, is the ratio between the critical speed and that which would be obtained if $\beta$ were set to zero (i.e. if shear effects were neglected). This ratio is shown as a function of $f \beta$ in Fig. 1.

When $f \beta=0$, the perturbation can be shown to be stationary with respect to the conducting body 1 , (i.e. $c_{1}^{*}=0$ ), whereas for non-zero $f \beta$, it moves over body 1 at a dimensionless speed $c_{1}^{*}$ which is generally small compared with the sliding speed, $V^{*}$. This velocity is also shown in Fig. 1. The sign of $f \beta$ determines the direction of the motion, positive $f \beta$ corresponding to negative $c_{1}^{*}$ and vice versa, but in other respects, the solutions obtained for equal positive and negative $f \beta$ are similar. It is therefore sufficient to present results for $f \beta>0$ in Fig. 1.

The parameter space of interest is restricted by the fact that $-0.5<\beta<0.5$ for materials with positive Poisson's ratio and the coefficient of friction, $f$, is unlikely to exceed unity in most practical situations. If $f \beta$ is small, it follows that the dimensionless velocity of the disturbance $c_{1}^{*}$ will also be small and an asymptotic approximation can be found to eqns. (23) and (26) which is

$\frac{V^{*}}{V_{0}^{*}}=1+\frac{10}{9}(f \beta)^{2}+O\left((f \beta)^{4}\right)$

$c_{1}^{*}=-\frac{4}{3} f \beta+O\left((\sigma \beta)^{3}\right)$

In view of the above considerations, $|f \beta|$ is unlikely to exceed 0.5 , at which $V^{*} / V_{0}^{*}=1.28$, indicating that

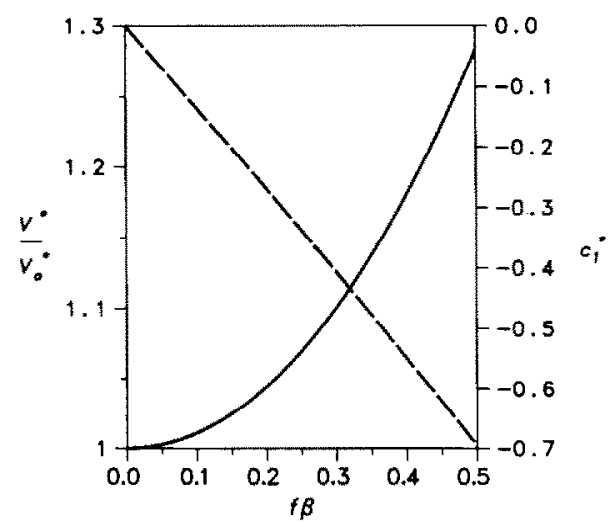

Fig. 1. The ratio between the critical speed and that obtained by neglecting shear effects $(-)$ and $c_{1}^{*}(---)$ when one material is a non-conductor. 
the neglect of shear effects would lead to an underestimate of the critical speed by $28 \%$. More realistic parameter values give much smaller errors - for example, with $\beta=0.25$ and $f=0.5$, we obtain an error of only $2 \%$. Also, since neglecting shear effects gives a lower prediction for critical speed, the approximation is a conservative one.

If more accurate results are desired, the asymptotic approximation of eqn. (28) gives an accuracy better than $\pm 0.5 \%$ in $V^{*}$ in the range $|f \beta|<0.5$.

\subsection{One material a poor conductor}

Of course, there are no non-conducting materials, but we might expect the above analysis to give a reasonable description for the case of a good conductor sliding on a poor conductor, i.e. for the case $K^{*} \ll 1$. However, the question arises as to how small $K^{*}$ must be in order that this approximation should be acceptable, with or without the inclusion of shear effects.

For this purpose, we must use the more general analysis leading to eqns. (16), (24) and (25) and the results depend on the five dimensionless parameters $f H_{1}, f \beta, K^{*}, k^{*}, \alpha^{*}$.

Properties of some common engineering materials are given in Table 1 . Notice that they show considerably more variability in $K$ and $k$ than in their ratio $K / k=\rho c_{\mathrm{p}}$ where $\rho, c_{\mathrm{p}}$ are respectively the density and the specific heat. We therefore replace the parameter pair $K^{*}, k^{*}$ by $K^{*},\left(\rho c_{\mathrm{p}}\right)^{*}$ where $\left(\rho c_{\mathrm{p}}\right)^{*}=K^{*} / k^{*}$.

Figure 2 shows the relation between $V^{*}$ and $f \beta$ for an interface between a friction material ( 7 in Table 1) and cast iron, for which $K^{*}=0.05,\left(\rho c_{p}\right)^{*}=0.62$, $\alpha^{*}=0.83, H_{1}=0.033$ and $\beta=-0.28$. An appropriate value of coefficient of friction, $f$, for this combination is 0.3 (see for example ref. 15) and the value $f H_{1}=0.0098$ was used. Also included in this figure are curves corresponding to different values of the ratio of conductivities, $K^{*}$, the other parameters remaining the same. The limiting case $K^{*}=0$ from the analysis of Section 2.2 is also included. It is clear that even the relatively small conductivity ratio $K^{*}=0.05$ results in an order of magnitude increase in critical speed over the case $K^{*}=0$ and there is also significant shear traction dependence, indicated by the slope of the curve. Notice that $V^{*}$ is plotted on a logarithmic scale, so a given deviation along this axis corresponds to the same percentage variation in critical speed. For a coefficient of friction of $0.3, f \beta=-0.0849$ and the shear effect increases the critical speed by an additional factor of 1.82 represented by the difference between the points A, B in Fig. 2.

Similar results for aluminum sliding on glass are presented in Fig. 3, for which the appropriate dimensionless parameters are $K^{*}=0.004,\left(\rho c_{\mathrm{p}}\right)^{*}=0.74$, $\alpha^{*}=0.32, \beta=-0.016$ and $H_{1}=0.33$. An appropriate value of coefficient of friction, $f$, for this combination is 0.1 (see for example ref. 16) and the value $f H_{1}=0.033$ was used. This time, since $K^{*}$ is lower, the error involved in using the non-conducting approximation is smaller and the shear traction effect is negligible (particularly bearing in mind the small value of $\beta$ ).

We conclude from these examples that the nonconducting approximation only gives a reasonable estimate of the critical speed if $K^{*}$ is exceedingly small. For example, non-conducting approximation typically underestimates the critical speed by an order of magnitude if $K^{*}>0.005$. We also note that significant errors will be introduced by neglecting the effect of shear tractions if $K^{*}>0.02$ unless $f \beta$ is very small. In particular, the effect of shear tractions should be taken into account in the prediction of critical speed for the important case of friction material sliding on cast iron.

\subsection{Materials with comparable conductivities}

When the two sliding materials have conductivities of the same order of magnitude, significantly higher critical speeds are obtained and in some cases the system is stable for all speeds.

For the special case of similar materials, $K^{*}=k^{*}=\alpha^{*}=1, \beta=0$, the critical speed depends only on $f H_{1}$. Equations (16), (24) and (25) can then be solved in closed form with the result

TABLE 1. Representative properties of some materials

\begin{tabular}{|c|c|c|c|c|c|c|}
\hline No. & Material & $\begin{array}{l}E \\
\left(\mathrm{~N} \mathrm{~m}^{-2} \times 10^{9}\right)\end{array}$ & $\nu$ & $\begin{array}{l}K \\
\left(\mathrm{~W} \mathrm{~m}^{1}{ }^{1}{ }^{\circ} \mathrm{C}^{\mathrm{t}}\right)\end{array}$ & $\begin{array}{l}k \\
\left(\mathrm{~m}^{2} \mathrm{~s}^{1} \times 10^{6}\right)\end{array}$ & $\begin{array}{l}\alpha \\
\left({ }^{\circ} \mathrm{C}\right. \\
{ }^{1} \times 10^{6}\end{array}$ \\
\hline 1 & Aluminum & 68 & 0.32 & 227 & 83 & 17 \\
\hline 2 & Copper & 120 & 0.33 & 381 & 101.9 & 17 \\
\hline 3 & Beryllium copper & 131 & 0.29 & 100 & 28.5 & 16.7 \\
\hline 4 & Gray cast iron & 103 & 0.26 & 50 & 12.86 & 12 \\
\hline 5 & Carbon steel & 207 & 0.3 & 47 & 13.27 & 12 \\
\hline 6 & Glass $^{\mathrm{a}}$ & 80 & 0.25 & 0.9 & 0.446 & 5.4 \\
\hline 7 & Friction material $^{\mathrm{a}}$ & 8.5 & 0.25 & 2.5 & 1.04 & 10 \\
\hline 8 & Carbon graphite & 10 & 0.25 & 12 & 8 & 5 \\
\hline
\end{tabular}

There is much more variation in properties of these types of solid than metals, variations occurring with production methods. 


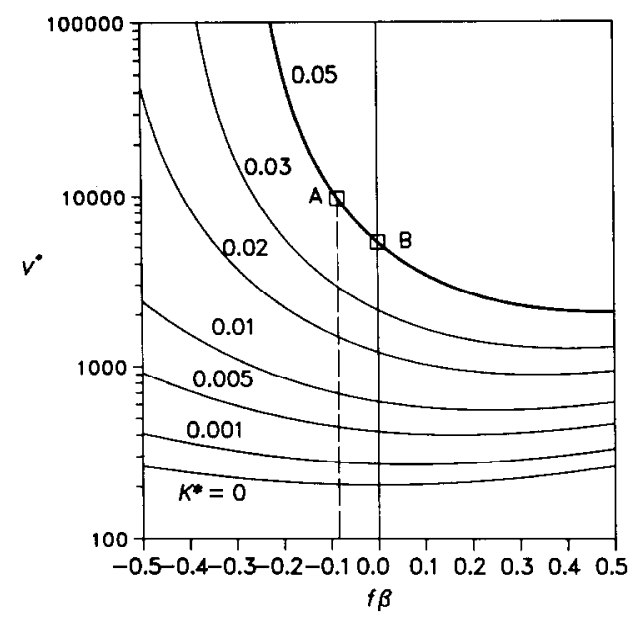

Fig. 2. The critical speed as a function of $f \beta$ for a friction material/cast iron interface. Results are presented for different values of $K^{*}$.

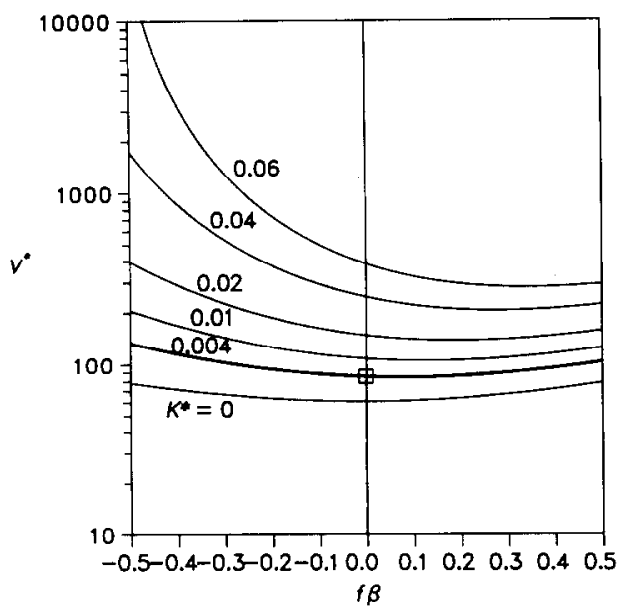

Fig. 3. The critical speed as a function of $f \beta$ for an aluminum/ glass interface. Results are presented for different values of $K^{*}$.

$V^{*}=\frac{8 f H_{1}}{4 f^{2} H_{1}^{2}-1}$

In particular, we note that there is no critical speed - i.e. the system is stable for all speeds - if $f H_{1}<0.5$. (Negative solutions of eqn. (30) are not physically meaningful, since the boundary condition (9) is based on the convention that $V>0$.) Since $H_{1}$ is generally less than unity, this implies that most cases involving similar materials will be unconditionally stable.

To examine the behavior of sliding pairs which differ only slightly in material properties, we consider the case where $\left(\rho c_{\mathrm{p}}\right)^{*}=\alpha^{*}=K^{*}=1$ and $f H_{1}$ takes various values. The corresponding curves are shown in Fig. 4. Notice in particular that for $f H_{1}<0.5$, the curves are all asymptotic to the line $f \beta=0$ and hence the system is unconditionally stable only for the value $f \beta=0$. Thus, in this case, the neglect of shear traction leads to a

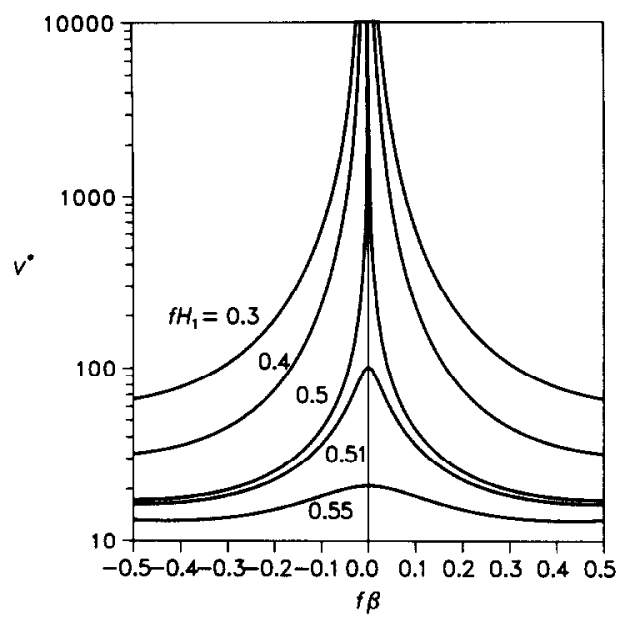

Fig. 4. The critical speed as a function of $f \beta$ for material pairs with $\left(\rho c_{\mathrm{p}}\right)^{*}=\alpha^{*}=K^{*}=1$. Results are presented for different values of $f H_{1}$.

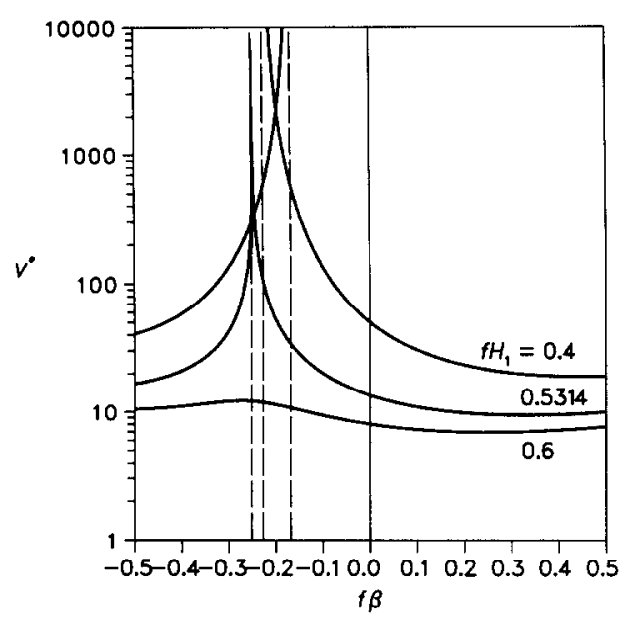

Fig. 5. The critical speed as a function of $f \beta$ for material pairs with $\left(\rho c_{\mathrm{p}}\right)^{*}=\alpha^{*}=1, K^{*}=0.6$. Results are presented for different values of $f H_{1}$.

totally erroneous (and non-conservative) prediction of the system behavior.

Figure 5 shows a similar set of results for the case where $K^{*}=0.6$, the other parameters remaining unchanged. We again find a critical value, $f H_{1}=0.5314$ at which the system can be unconditionally stable, but in this case, the lack of symmetry in thermal conductivity displaces the asymptote to the value $f \beta=-0.2516$. We also find that for $f H_{1}<0.5314$, the asymptotes for the two branches of the curve are different, both being closer to $f \beta=0$ than the asymptote for the critical value. The two branches of the curve therefore cross at a finite value of $V^{*}$ and the system is therefore unconditionally stable only at the isolated point $f H_{1}=0.5314$, $f \beta=-0.2516$. 


\section{Conclusions}

The above results demonstrate that there are ranges of parameter values in which significant error will be introduced if the shear effect is neglected in predicting critical speeds for thermoelastic instability. The effect is particularly significant when the two materials have thermal properties - particularly thermal conductivity - of the same order of magnitude. In the special case where the thermal properties are identical, unconditionally stable behavior is predicted when the shear effect is neglected, for typical coefficients of friction and material properties. By contrast, inclusion of the deformation due to shear tractions, leads to bounded values of critical speed, showing that the simpler solution is non-conservative.

When the thermal conductivities of the two materials are very different, it is traditional to adopt a simpler analysis in which the less good conductor is replaced by an insulator. Results are presented showing that this simplification typically underestimates the critical speed by at least an order of magnitude, if the ratio of conductivities $K^{*}$ is greater than 0.005 .

\section{References}

1 J. R. Barber, The influence of thermal expansion on the friction and wear process, Wear, 10 (1967) 155-159.

2 J. R. Barber, Thermoelastic instabilities in the sliding of conforming solids, Proc. R. Soc. London, Ser. A, 312 (1969) 381-394.
3 J. L. van Swaaij, Thermal damage to railway wheels, Inst. Mech. Eng., Int. Conf. on Railway Braking, York, 1979, Institution of Mechanical Engineers, London, p. 95.

4 G. G. Hewitt and C. Musial, The search for improved wheel materials, Inst. Mech. Eng., Int. Conf. on Railway Braking, York, 1979, Institution of Mechanical Engineers, London, p. 101.

5 T. A. Dow and R. D. Stockwell, Experimental verification of thermoelastic instabilities in sliding contact, Trans. ASME, Ser. F, 261 (1977) 359-364.

6 J. P. Netzcl, Obscrvations of thermoelastic instability in mechanical face seals, Wear, 59 (1980) 135-148.

7 F. E. Kennedy, Jr., and J. N. Grim, Observation of contact conditions in mechanical face seals, ASLE Trans., 27 (1984) 122-128.

8 T. A. Dow and R. A. Burton, Thermoelastic instability of sliding contact in the absence of wear, Wear, 19 (1972) 315-328.

9 R. A. Burton, V. Nerlikar and S. R. Kilaparti, Thermoelastic instability in a seal-like configuration, Wear, 24 (1973) 177-188.

10 E. A. Kuznetsov, Superposition principle in the solution of contact problems with allowance for friction, Sov. Appl. Mech., 14 (1978) 494-498.

11 S. R. Heckmann and R. A. Burton, Effects of shear and wear on instabilities caused by frictional heating in a seallike configuration, ASLE Trans., 20 (1977) 71-78.

12 J. Dundurs, Discussion on edge-bonded dissimilar orthogonal elastic wedges under normal and shear loading, ASME $J$. Appl. Mech., 36 (1969) 650-652.

13 J. R. Barber, Elasticity, Kluwer Academic Publishers, Dordrecht, 1992.

14 D. A. Hills and J. R. Barber, Steady motion of an insulating rigid flat-ended punch over a thermally conducting half-plane, Wear, 102 (1985) 15-22.

15 A. E. Anderson, S. Gratch and H. P. Hayes, A New Laboratory Friction and Wear Test for the Characterization of Brake Linings, SAE 670079.

16 R. A. Burton, Thermal deformation in frictionally beated contact, Wear, 59 (1980) 1-20. 